\title{
Hashing on Nonlinear Manifolds
}

\author{
Fumin Shen, Chunhua Shen, Qinfeng Shi, Anton van den Hengel, Zhenmin Tang, Heng Tao Shen
}

\begin{abstract}
Learning based hashing methods have attracted considerable attention due to their ability to greatly increase the scale at which existing algorithms may operate. Most of these methods are designed to generate binary codes preserving the Euclidean similarity in the original space. Manifold learning techniques, in contrast, are better able to model the intrinsic structure embedded in the original high-dimensional data. The complexities of these models, and the problems with out-ofsample data, have previously rendered them unsuitable for application to large-scale embedding, however.

In this work, how to learn compact binary embeddings on their intrinsic manifolds is considered. In order to address the abovementioned difficulties, an efficient, inductive solution to the outof-sample data problem, and a process by which non-parametric manifold learning may be used as the basis of a hashing method is proposed. The proposed approach thus allows the development of a range of new hashing techniques exploiting the flexibility of the wide variety of manifold learning approaches available. It is particularly shown that hashing on the basis of t-SNE [41] outperforms state-of-the-art hashing methods on large-scale benchmark datasets, and is very effective for image classification with very short code lengths. The proposed hashing framework is shown to be easily improved, for example, by minimizing the quantization error with learned orthogonal rotations. In addition, a supervised inductive manifold hashing framework is developed by incorporating the label information, which is shown to greatly advance the semantic retrieval performance.
\end{abstract}

Index Terms-Hashing, binary code learning, manifold learning, image retrieval.

\section{INTRODUCTION}

One key challenge in many large scale image data based applications is how to index and organize the data accurately, but also efficiently. Various hashing techniques have attracted considerable attention in computer vision, information retrieval and machine learning [11], [13], [27], [28], [43], [46], and seem to offer great promise towards this goal. Hashing methods aim to encode documents or images as a set of short binary codes, while maintaining aspects of the structure of the original data (e.g., similarities between data points). The advantage of these compact binary representations is that pairwise comparisons may be carried out extremely efficiently

F. Shen is with School of Computer Science and Engineering, University of Electronic Science and Technology of China, Chengdu 611731, China (e-mail: fumin.shen@gmail.com). Part of this work was done when the first author was visiting The University of Adelaide.

C. Shen, Q. Shi and A. van den Hengel are with School of Computer Science at The University of Adelaide, SA 5005, Australia (e-mail: \{chunhua.shen, javen.shi, anton.vandenhengel\}@adelaide.edu.au). C. Shen and A. van den Hengel are also with Australian Centre for Robotic Vision. Correspondence should be addressed to C. Shen.

Z. Tang is with the School of Computer Science and Technology, Nanjing University of Science and Technology, Nanjing 210094, P.R. China (e-mail: tzm.cs@mail.njust.edu.cn).

H. T. Shen is with School of Information Technology and Electrical Engineering, The University of Queensland, Australia (E-mail: shenht@itee.uq.edu.au). in the Hamming space. This means that many algorithms which are based on such pairwise comparisons can be made more efficient, and applied to much larger datasets. Due to the flexibility of hash codes, hashing techniques can be applied in many ways. one can, for example, efficiently perform similarity search by exploring only those data points falling into the close-by buckets to the query by the Hamming distance, or use the binary representations for other tasks like image classification.

Locality sensitive hashing (LSH) [11] is one of the most well-known data-independent hashing methods, and generates hash codes based on random projections. With the success of LSH, random hash functions have been extended to several similarity measures, including $p$-norm distances [7], the Mahalanobis metric [25], and kernel similarity [24], [35]. However, the methods belonging to the LSH family normally require relatively long hash codes and several hash tables to achieve both high precision and recall. This leads to a larger storage cost than would otherwise be necessary, and thus limits the sale at which the algorithm may be applied.

Data-dependent or learning-based hashing methods have been developed with the goal of learning more compact hash codes. Directly learning binary embeddings typically results in an optimization problem which is very difficult to solve, however. Relaxation is often used to simplify the optimization (e.g., [3], [43]). As in LSH, the methods aim to identify a set of hyperplanes, but now these hyperplanes are learned, rather than randomly selected. For example, PCAH [43], SSH [43], and iterative quantization (ITQ) [13] generate linear hash functions through simple principal component analysis (PCA) projections, while LDAhash [3] is based on Linear Discriminant Analysis (LDA). Extending this idea, there are also methods which learn hash functions in a kernel space, such as binary reconstructive embeddings (BRE) [23], random maximum margin hashing (RMMH) [22] and kernel-based supervised hashing (KSH) [30]. In a departure from such methods, however, spectral hashing ( $\mathrm{SH}$ ) [46], one of the most popular learning-based methods, generates hash codes by solving the relaxed mathematical program that is similar to the one in Laplacian eigenmaps [1].

Embedding the original data into a low dimensional space while simultaneously preserving the inherent neighborhood structure is critical for learning compact, effective hash codes. In general, nonlinear manifold learning methods are more powerful than linear dimensionality reduction techniques, as they are able to more effectively preserve the local structure of the input data without assuming global linearity [38]. The geodesic distance on a manifold has been shown to outperform the Euclidean distance in the high-dimensional space for image retrieval [16], for example. Figure 2 demonstrates that searching using either the Euclidean or Hamming distance 


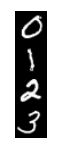

(a) Queries

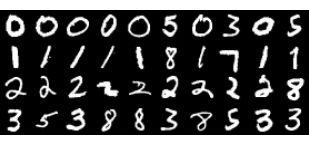

(b) $\ell_{2}$ dist. on $784 \mathrm{D}$

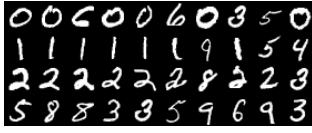

(c) LSH with 128-bits

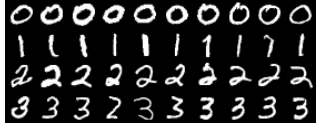

(d) $\ell_{2}$ dist. on embeded $48 \mathrm{D}$

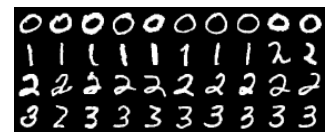

(e) Hamm. dist. with 48-bits

Fig. 2: Top 10 retrieved digits for 4 queries (a) on a subset of MNIST with 300 samples. Search is conducted in the original feature space (b, c) and nonlinear embedding space by t-SNE [41] (d, e) using Euclidean distance (b, d) and Hamming distance (c, e).

after nonlinear embedding results in more semantically accurate neighbors than the same search in the original feature space, and thus that low-dimensional embedding may actually improve retrieval or classification performance. However, the only widely used nonlinear embedding method for hashing is Laplacian eigenmaps (LE) (e.g., in [46], [48], [31]). Other effective manifold learning approaches (e.g., Locally Linear Embedding (LLE) [36], Elastic Embedding (EE) [4] or tDistributed Stochastic Neighbor Embedding (t-SNE) [41]) have rarely been explored for hashing. Very recently, the authors of [20] choose to jointly minimize the LLE embedding error and the quantization loss with an orthogonal rotation.

One problem hindering the use of manifold learning for hashing is that these methods do not directly scale to large datasets. For example, to construct the neighborhood graph (or pairwise similarity matrix) in these algorithms for $n$ data points is $O\left(n^{2}\right)$ in time, which is intractable for large datasets. The second problem is that they are typically non-parametric and thus cannot efficiently solve the critical out-of-sample extension problem. This fundamentally limits their application to hashing, as generating codes for new samples is an essential part of the problem. One of the widely used solutions for the methods involving spectral decomposition (e.g., LLE, LE and isometric feature mapping (ISOMap) [39]) is the Nyström extension [2], [38], which solves the problem by learning eigenfunctions of a kernel matrix. As mentioned in [46], however, this is impractical for large-scale hashing since the Nyström extension is as expensive as doing exhaustive nearest neighbor search $(O(n))$. A more significant problem, however, is the fact that the Nyström extension cannot be directly applied to non-spectral manifold learning methods such as $\mathrm{t}$-SNE.

In order to address the out-of-sample extension problem, this study proposes a new non-parametric regression approach which is both efficient and effective. This method allows rapid assignment of new codes to previously unseen data in a manner which preserves the underlying structure of the manifold. Having solved the out-of-sample extension problem, a method by which a learned manifold may be used as the basis for a binary encoding is introduced. This method is designed so as to generate encodings which reflect the geodesic distances along such manifolds. On this basis, a range of new embedding approaches based on a variety of manifold learning methods are developed. The best performing of these is based on manifolds identified through t-SNE, which has been shown to be effective in discovering semantic manifolds amongst the set of all images [41].
Given the computational complexity of many manifold learning methods, it is shown in this work that it is possible to learn the manifold on the basis of a small subset of the data $\mathbf{B}$ (with size $m \ll n$ ), and subsequently to inductively insert the remainder of the data, and any out-of-sample data, into the embedding in $O(m)$ time per point. This process leads to an embedding method labelled as Inductive ManifoldHashing (IMH) which is shown to outperform state-of-the-art methods on several large scale datasets both quantitatively and qualitatively.

As an extension, this study shows that the proposed IMH framework can be naturally improved by minimizing the quantization error, for example, through orthogonal rotations. Significant performance gains are achieved by this simple step, as shown in Section $\mathrm{V}$. Based on supervised subspace learning, this study also presents a supervised inductive manifold hashing framework (IMHs) (Section VI), which is shown to significantly advance the semantic retrieval performance of IMH.

The rest of this paper is organized as follows. In Section III. some representative hashing methods related are briefly reviewed. Section III describes the proposed Inductive ManifoldHashing framework, followed by the experimental results in Section IV] The IMH method is further shown to be improved by learned orthogonal rotations in Section V. Section VI introduces the supervised extension of the inductive manifold hashing framework based on supervised subspace learning.

This paper is an extended version of the work previously published in [37]. Major improvements over [37] include the quantization error minimization by learned rotations (Section $\mathrm{VP}$ ) and the supervised extension (Section VI).

\section{RELATED WORK}

Learning based or data-dependent hashing has achieved considerable attention recently in computer vision, machine learning and information retrieval community. Many hashing methods have been proposed by applying different learning algorithms, including unsupervised methods [13], [31], [46], [48], and (semi-)supervised methods [23][30][28][43]. In this section, some representative unsupervised hashing methods related to the proposed method are briefly reviewed. 


\section{A. Spectral Hashing}

Weiss et al. [46] formulated the spectral hashing ( $\mathrm{SH})$ problem as

$$
\begin{aligned}
& \min _{\mathbf{Y}} \sum_{\mathbf{x}_{i}, \mathbf{x}_{j} \in \mathbf{X}} \mathrm{w}\left(\mathbf{x}_{i}, \mathbf{x}_{j}\right)\left\|\mathbf{y}_{i}-\mathbf{y}_{j}\right\|^{2} \\
& \text { s.t. } \mathbf{Y} \in\{-1,1\}^{n \times r}, \mathbf{Y}^{\top} \mathbf{Y}=n \mathbf{I}, \mathbf{Y}^{\top} \mathbf{1}=0 .
\end{aligned}
$$

Here $\mathbf{y}_{i} \in\{-1,1\}^{r}$, the $i$ th row in $\mathbf{Y}$, is the hash code one wants to learn for $\mathbf{x}_{i} \in \mathbf{R}^{d}$, which is one of the $n$ data points in the training data set $\mathbf{X}$. W $\in \mathbf{R}^{n \times n}$ with $\mathbf{W}_{i j}=\mathrm{w}\left(\mathbf{x}_{i}, \mathbf{x}_{j}\right)=\exp \left(-\left\|\mathbf{x}_{i}-\mathbf{x}_{j}\right\|^{2} / \sigma^{2}\right)$ is the graph affinity matrix, where $\sigma$ is the bandwidth parameter. $\mathbf{I}$ is the identity matrix. The last two constraints force the learned hash bits to be uncorrelated and balanced, respectively. By removing the first constraint (i.e., spectral relaxation [46]), $\mathbf{Y}$ can be easily obtained by spectral decomposition on the Laplcaian matrix $\mathbf{L}=\mathbf{D}-\mathbf{W}$, where $\mathbf{D}=\operatorname{diag}(\mathbf{W} \mathbf{1})$ and $\mathbf{1}$ is the vector with all ones. However, constructing $\mathbf{W}$ is $O\left(d n^{2}\right)$ (in time) and calculating the Nyström extension for a new point is $O(r n)$, which are both intractable for large datasets. It is assumed in SH [46], therefore, that the data are sampled from a uniform distribution, which leads to a simple analytical eigenfunction solution of 1-D Laplacians. However, this strong assumption is not true in practice and the manifold structure of the original data are thus destroyed [31].

SH was extended into the tangent space in [6], however, based on the same uniform assumption. [6] also proposed a non-Euclidean SH algorithm based on nonlinear clustering, which is $O\left(n^{3}\right)$ for training and $O(m+n / m)$ for testing. Weiss et al. [45] then improved SH by expanding the codes to include the outer-product eigenfunctions instead of only single-dimension eigenfunctions in $\mathrm{SH}$, i.e., multidimensional spectral hashing (MDSH).

\section{B. Graph based hashing}

To efficiently solve problem (1), anchor graph hashing (AGH) [31] approximated the affinity matrix $\mathbf{W}$ by the lowrank matrix $\hat{\mathbf{W}}=\mathbf{Z} \Lambda^{-1} \mathbf{Z}$, where $\mathbf{Z} \in \mathbf{R}^{n \times m}$ is the normalized affinity matrix (with $k$ non-zeros in each row) between the training samples and $m$ anchors (generated by $K$-means), and $\Lambda^{-1}$ normalizes $\hat{\mathbf{W}}$ to be doubly stochastic. Then the desired hash functions may be efficiently identified by binarizing the Nyström eigenfunctions [2] with the approximated affinity matrix $\hat{\mathbf{W}}$. AGH is thus efficient, in that it has linear training time and constant search time, but as is the case for SH [46], the generalized eigenfunction is derived only for the Laplacian eigenmaps embedding.

Different from SH and AGH, Locally Linear Hashing (LLH [20]) constructs the graph affinity by locality-sensitive sparse coding to better capture the local linearity of manifolds. With the obtained affinity matrix, LLH formulates hashing as a joint optimization problem of LLE embedding error and quantization loss.

\section{Self-Taught Hashing}

Self-taught hashing (STH) [48] addressed the out-of-sample problem by a novel way: hash functions are obtained by training a support vector machine (SVM) classifier for each bit using the pre-learned binary codes as class labels. The binary codes were learned by directly solving (1) with a cosine similarity function. This process has prohibitive computational and memory costs, however, and training the SVM can be very time consuming for dense data. Very recently, this idea was applied in collaboration with graph cuts to the binary coding problems to bypass continuous relaxation [10].

\section{THE PROPOSED METHOD}

\section{A. Inductive learning for hashing}

Assuming that one has the manifold-based embedding $\mathbf{Y}:=$ $\left\{\mathbf{y}_{1}, \mathbf{y}_{2}, \cdots, \mathbf{y}_{n}\right\}$ for the entire training data $\mathbf{X}:=\left\{\mathbf{x}_{1}, \mathbf{x}_{2}\right.$, $\left.\cdots, \mathbf{x}_{n}\right\}$. Given a new data point $\mathbf{x}_{q}$, one aims to generate an embedding $\mathbf{y}_{q}$ which preserves the local neighborhood relationships among its neighbors $\mathcal{N}_{k}\left(\mathbf{x}_{q}\right)$ in $\mathbf{X}$. The following simple objective is utilized:

$$
\mathcal{C}\left(\mathbf{y}_{q}\right)=\sum_{i=1}^{n} \mathrm{w}\left(\mathbf{x}_{q}, \mathbf{x}_{i}\right)\left\|\mathbf{y}_{q}-\mathbf{y}_{i}\right\|^{2}
$$

where

$$
\mathrm{w}\left(\mathbf{x}_{q}, \mathbf{x}_{i}\right)=\left\{\begin{array}{cl}
\exp \left(-\left\|\mathbf{x}_{q}-\mathbf{x}_{i}\right\|^{2} / \sigma^{2}\right), & \text { if } \mathbf{x}_{i} \in \mathcal{N}_{k}\left(\mathbf{x}_{q}\right), \\
0 & \text { otherwise. }
\end{array}\right.
$$

Minimizing (2) naturally uncovers an embedding for the new point on the basis of its nearest neighbors on the lowdimensional manifold initially learned on the base set. That is, in the low-dimensional space, the new embedded location for the point should be close to those of the points close to it in the original space.

Differentiating $\mathcal{C}\left(\mathbf{y}_{q}\right)$ with respect to $\mathbf{y}_{q}$, one obtains

$$
\left.\frac{\partial \mathcal{C}\left(\mathbf{y}_{q}\right)}{\mathbf{y}_{q}}\right|_{\mathbf{y}_{q}=\mathbf{y}_{q}^{\star}}=2 \sum_{i=1}^{n} \mathrm{w}\left(\mathbf{x}_{q}, \mathbf{x}_{i}\right)\left(\mathbf{y}_{q}^{\star}-\mathbf{y}_{i}\right)=0,
$$

which leads to the optimal solution

$$
\mathbf{y}_{q}^{\star}=\frac{\sum_{i=1}^{n} \mathrm{w}\left(\mathbf{x}_{q}, \mathbf{x}_{i}\right) \mathbf{y}_{i}}{\sum_{i=1}^{n} \mathrm{w}\left(\mathbf{x}_{q}, \mathbf{x}_{i}\right)} .
$$

Equation (4) provides a simple inductive formulation for the embedding: produce the embedding for a new data point by a (sparse) locally linear combination of the base embeddings.

The proposed approach here is inspired by Delalleau et al. [8], where they have focused on non-parametric graphbased learning in semi-supervised classification. The aim of this study here is completely different: the present work tries to scale up the manifold learning process for hashing in an unsupervised manner.

The resulting solution (4) is consistent with the basic smoothness assumption in manifold learning, that close-by data points lie on or close to a locally linear manifold [36], [39], [1]. This local-linearity assumption has also been widely used in semi-supervised learning [8], [47], image coding [44], and similar. This paper proposes to apply this assumption to hash function learning.

However, as aforementioned, (4) does not scale well for both computing $\mathbf{Y}\left(O\left(n^{2}\right)\right.$ e.g., for LE) and out-of-sample extension $(O(n))$, which is intractable for large scale tasks. Next, it is shown that the following prototype algorithm is able to approximate $\mathbf{y}_{q}$ using only a small base set well. 


\section{B. The prototype algorithm}

This prototype algorithm is based on entropy numbers defined below.

Definition 1 (Entropy numbers[18]). Given any $Y \subseteq \mathbb{R}^{r}$ and $m \in \mathbb{N}$, the $m$-th entropy number $\epsilon_{m}(Y)$ of $Y$ is defined as

$$
\epsilon_{m}(Y):=\inf \{\epsilon>0 \mid \mathcal{N}(\epsilon, Y,\|\cdot-\cdot\|) \leq m\},
$$

where $\mathcal{N}$ is the covering number. This means $\epsilon_{m}(Y)$ is the smallest radius that $Y$ can be covered by less or equal to $m$ balls.

Inspired by Theorem 27 of [18], a prototype algorithm is constructed below. One can use $m$ balls to cover $Y$, thus obtain $m$ disjoint nonempty subsets $Y_{1}, Y_{2}, \cdots, Y_{m}$ such that for any $\epsilon>\epsilon_{m}(Y), \forall j \in\{1, \cdots, m\}, \exists \mathbf{c}_{j} \in \mathbb{R}^{r}$, s.t. $\forall \mathbf{y} \in Y_{j}, \| \mathbf{c}_{j}-$ $\mathbf{y} \| \leq \epsilon$ and $\bigcup_{j=1}^{m} Y_{j}=Y$. One can see that each $Y_{j}$ naturally forms a cluster with the center $\mathbf{c}_{j}$ and the index set $I_{j}=$ $\left\{i \mid \mathbf{y}_{i} \in Y_{j}\right\}$.

Let $\alpha_{i}=\frac{\mathrm{w}\left(\mathbf{x}_{q}, \mathbf{x}_{i}\right)}{\sum_{j=1}^{n} \mathrm{w}\left(\mathbf{x}_{q}, \mathbf{x}_{j}\right)}$ and $C_{j}=\sum_{i \in I_{j}} \alpha_{i}$. For each cluster index set $I_{j}, j=1, \cdots, m, \ell_{j}=\left\lfloor m C_{j}+1\right\rfloor$ many indices are randomly drawn from $I_{j}$ proportional to their weight $\alpha_{i}$. That is, for $\mu \in\left\{1, \cdots, \ell_{j}\right\}$, the $\mu$-th randomly drawn index $u_{j, \mu}$,

$$
\operatorname{Pr}\left(u_{j, \mu}=i\right)=\frac{\alpha_{i}}{C_{j}}, \forall j \in\{1, \cdots, m\} .
$$

$\hat{\mathbf{y}}_{q}$ is constructed as

$$
\hat{\mathbf{y}}_{q}=\sum_{j=1}^{m} \frac{C_{j}}{\ell_{j}} \sum_{\mu=1}^{\ell_{j}} \mathbf{y}_{u_{j, \mu}} .
$$

Lemma 2. There is at most $2 m$ many unique $\mathbf{y}_{u_{j, \mu}}$ in $\hat{\mathbf{y}}_{q}$.

Proof: $\sum_{j=1}^{m} \ell_{j} \leq \sum_{j=1}^{m}\left(m C_{j}+1\right)=2 m$.

The following lemma shows that through the prototype algorithm the mean is preserved and variance is small.

Lemma 3. The following holds

$$
\mathrm{E}\left[\hat{\mathbf{y}}_{q}\right]=\mathbf{y}_{q}, \operatorname{Var}\left(\hat{\mathbf{y}}_{q}\right) \leq \frac{\epsilon^{2}}{m} .
$$

Proof:

$$
\begin{aligned}
& \mathrm{E}\left[\hat{\mathbf{y}}_{q}\right]=\mathrm{E}\left[\sum_{j=1}^{m} \frac{C_{j}}{\ell_{j}} \sum_{\mu=1}^{\ell_{j}} \mathbf{y}_{u_{j, \mu}}\right]=\sum_{j=1}^{m} \frac{C_{j}}{\ell_{j}} \sum_{\mu=1}^{\ell_{j}} \mathrm{E}\left[\mathbf{y}_{u_{j, \mu}}\right] \\
& =\sum_{j=1}^{m} \frac{C_{j}}{\ell_{j}} \sum_{\mu=1}^{\ell_{j}} \sum_{i \in I_{j}} \frac{\alpha_{i}}{C_{j}} \mathbf{y}_{i}=\sum_{j=1}^{m} \sum_{i \in I_{j}} \alpha_{i} \mathbf{y}_{i}=\mathbf{y}_{q} . \\
& \operatorname{Var}\left(\hat{\mathbf{y}}_{q}\right)=\sum_{j=1}^{m} \sum_{\mu=1}^{\ell_{j}} \operatorname{Var}\left(\frac{C_{j}}{\ell_{j}} \mathbf{y}_{u_{j, \mu}}\right) \leq \sum_{j=1}^{m} \frac{C_{j}^{2}}{\ell_{j}^{2}} \sum_{\mu=1}^{\ell_{j}} \epsilon^{2} \\
& =\sum_{j=1}^{m} \frac{C_{j}^{2}}{\ell_{j}} \epsilon^{2} \leq \sum_{j=1}^{m} \frac{C_{j}^{2}}{m C_{j}} \epsilon^{2}=\frac{\sum_{j=1}^{m} C_{j}^{2}}{m} \epsilon^{2}=\frac{\epsilon^{2}}{m} .
\end{aligned}
$$

Theorem 4. For any even number $n^{\prime} \leq n$. If Prototype Algorithm uses $n^{\prime}$ many non-zero $\mathbf{y} \in Y$ to express $\hat{\mathbf{y}}_{q}$, then

$$
\operatorname{Pr}\left[\left\|\hat{\mathbf{y}}_{q}-\mathbf{y}_{q}\right\| \geq t\right]<\frac{2\left(\epsilon_{\frac{n^{\prime}}{2}}(Y)\right)^{2}}{n^{\prime} t^{2}} .
$$

Proof: Via Chebyshev's inequality and Lemma 3, one gets

$$
\operatorname{Pr}\left(\left\|\hat{\mathbf{y}}_{q}-\mathbf{y}_{q}\right\| \geq k \sqrt{\operatorname{Var}\left(\hat{\mathbf{y}}_{q}\right)}\right) \leq \frac{1}{k^{2}} .
$$

Let $t=k \sqrt{\operatorname{Var}\left(\hat{\mathbf{y}}_{q}\right)}$ and $\epsilon \rightarrow \epsilon_{\frac{n^{\prime}}{2}}(Y)$ yields the theorem.

Corollary 5. For an even number $n^{\prime}$, any $\epsilon>\epsilon_{\frac{n^{\prime}}{2}}(Y)$, any $\delta \in(0,1)$ and any $t>0$, if $n^{\prime} \geq \frac{2 \epsilon^{2}}{\delta t^{2}}$, then with probability at least $1-\delta$,

$$
\left\|\hat{\mathbf{y}}_{q}-\mathbf{y}_{q}\right\|<t .
$$

Proof: Via Theorem 4, for $\epsilon>\epsilon_{\frac{n^{\prime}}{2}}(Y), \operatorname{Pr}\left[\left\|\hat{\mathbf{y}}_{q}-\mathbf{y}_{q}\right\| \geq\right.$ $t]<\frac{2 \epsilon^{2}}{n^{\prime} t^{2}}$. Let $\delta \geq \frac{2 \epsilon^{2}}{n^{\prime} t^{2}}$, the following holds $n^{\prime} \geq \frac{2 \epsilon^{2}}{\delta t^{2}}$.

The quality of the approximation depends on $\epsilon_{\frac{n^{\prime}}{2}}(Y)$ and $n^{\prime}$. If data has strong clustering pattern, i.e. data within each cluster are very close to cluster center, one will have small $\epsilon_{\frac{n^{\prime}}{2}}(Y)$, hence better approximation. Likewise, the bigger $n^{\prime}$ is, the better approximation is.

\section{Approximation of the prototype algorithm}

For a query point $\mathbf{x}_{q}$, the prototype algorithm samples from clusters and then construct $\hat{\mathbf{y}}_{q}$. The clusters can be obtained via clustering algorithm such as K-means. For each cluster, the higher $C_{j}=\sum_{i \in I_{j}} \alpha_{i}$, the more draws are made. At least one draw is made from each cluster. Since the $n$ could be potentially massive, it is impractical to rank (or compute and keep a few top ones) $\alpha_{i}$ with in each cluster. Moreover, $\mathrm{w}\left(\mathbf{x}_{q}, \mathbf{x}_{j}\right)$ depends on $\mathbf{x}_{q}$ - for a different query point $\mathbf{x}_{q^{\prime}}$, $\mathrm{w}\left(\mathbf{x}_{q^{\prime}}, \mathbf{x}_{i}\right)$ may be very smaller even if $\mathrm{w}\left(\mathbf{x}_{q}, \mathbf{x}_{i}\right)$ is high. Thus one needs to consider the entire $X$ instead of a single $\mathbf{x}_{q}$.

Let $\alpha_{i}\left(\mathbf{x}_{q}\right)=\frac{\mathrm{w}\left(\mathbf{x}_{q}, \mathbf{x}_{i}\right)}{\sum_{j=1}^{n} \mathrm{w}\left(\mathbf{x}_{q}, \mathbf{x}_{j}\right)}$. Ideally, for each cluster, one wants to select the $\mathbf{y}_{i}$ that has high overall weight $O_{i}=\sum_{\mathbf{x}_{q} \in X} \alpha_{i}\left(\mathbf{x}_{q}\right)$. For large scale $X$, the reality is that one does not have access to $\mathrm{w}\left(\mathbf{x}, \mathbf{x}^{\prime}\right)$ for all $\mathbf{x}, \mathbf{x}^{\prime} \in X$. Only limited information is available such as cluster centers $\left\{\mathbf{c}_{j}, j \in\{1, \cdots, m\}\right\}$ and $\mathrm{w}\left(\mathbf{c}_{j}, \mathbf{x}\right), \mathbf{x} \in X$. Fortunately, the clustering result gives useful information about $O_{i}$. The cluster centers $\left\{\mathbf{c}_{j}, j \in\{1, \cdots, m\}\right\}$ have the largest overall weight w.r.t the points from their own cluster, i.e. $\sum_{i \in I_{j}} \mathrm{w}\left(\mathbf{c}_{j}, \mathbf{x}_{i}\right)$. This suggests one should select all cluster centers to express $\hat{\mathbf{y}}_{q}$. For a base set $B$, and any query point $\mathbf{x}_{q}$, the embedding is predicted as

$$
\hat{\mathbf{y}}_{q}=\frac{\sum_{\mathbf{x} \in B} \mathrm{w}\left(\mathbf{x}_{q}, \mathbf{x}\right) \mathbf{y}}{\sum_{\mathbf{x} \in B} \mathrm{w}\left(\mathbf{x}_{q}, \mathbf{x}\right)} .
$$

Following many methods in the area (e.g., [46], [31]), the general inductive hash function is formulated by binarizing the low-dimensional embedding

$$
h(\mathbf{x})=\operatorname{sgn}\left(\frac{\sum_{j=1}^{m} \mathrm{w}\left(\mathbf{x}, \mathbf{c}_{j}\right) \mathbf{y}_{j}}{\sum_{j=1}^{m} \mathrm{w}\left(\mathbf{x}, \mathbf{c}_{j}\right)}\right),
$$




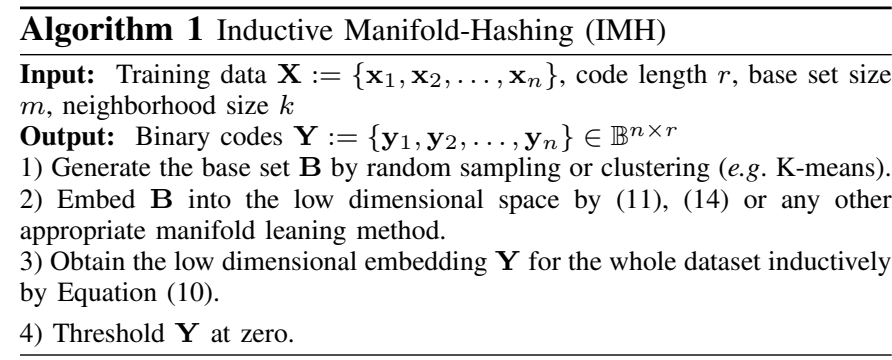

where $\operatorname{sgn}(\cdot)$ is the sign function and $\mathbf{Y}_{\mathbf{B}}:=\left\{\mathbf{y}_{1}, \mathbf{y}_{2}, \cdots\right.$, $\left.\mathbf{y}_{m}\right\}$ is the embedding for the base set $\mathbf{B}:=\left\{\mathbf{c}_{1}, \mathbf{c}_{2}, \cdots, \mathbf{c}_{m}\right\}$, which is the cluster centers obtained by K-means. Here the embedding $\mathbf{y}_{i}$ are assumed to be centered on the origin. The proposed hashing method is termed Inductive ManifoldHashing (IMH). The inductive hash function provides a natural means for generalization to new data, which has a constant $O(d m+r k)$ time. With this, the embedding for the training data becomes

$$
\mathbf{Y}=\overline{\mathbf{W}}_{\mathbf{X B}} \mathbf{Y}_{\mathbf{B}}
$$

where $\overline{\mathbf{W}}_{\mathbf{X B}}$ is defined such that $\overline{\mathbf{W}}_{i j}=\frac{\mathrm{w}\left(\mathbf{x}_{i}, \mathbf{c}_{j}\right)}{\sum_{i=1}^{m} \mathrm{w}\left(\mathbf{x}_{i}, \mathbf{c}_{j}\right)}$, for $\mathbf{x}_{i}$ $\in \mathbf{X}, \mathbf{c}_{j} \in \mathbf{B}$.

Although the objective function (2) is formally related to $\mathrm{LE}$, it is general in preserving local similarity. The embeddings $\mathbf{Y}_{\mathbf{B}}$ can be learned by any appropriate manifold learning method which preserves the similarity of interest in the low dimensional space. Several other embedding methods are empirically evaluated in Section III-F Actually, as will be shown, some manifold learning methods (e.g., t-SNE described in Section III-D can be better choices for learning binary codes, although LE has been widely used. Two methods for learning $\mathbf{Y}_{\mathbf{B}}$ will be discussed in the sequel.

The Inductive Manifold-Hashing framework is summarized in Algorithm 1. Note that the computational cost is dominated by K-means in the first step, which is $O(d m n l)$ in time (with $l$ the number of iterations). Considering that $m$ (normally a few hundreds) is much less than $n$, and is a function of manifold complexity rather than the volume of data, the total training time is linear in the size of training set. If the embedding method is LE, for example, then using IMH to compute $\mathbf{Y}_{\mathbf{B}}$ requires constructing the small affinity matrix $\mathbf{W}_{\mathbf{B}}$ and solving $r$ eigenvectors of the $m \times m$ Laplacian matrix $\mathbf{L}_{\mathbf{B}}$ which is $O\left(d m^{2}+r m\right)$. Note that in step 3 , to compute $\overline{\mathbf{W}}_{\mathbf{X B}}$, one needs to compute the distance matrix between $\mathbf{B}$ and $\mathbf{X}$, which is a natural output of K-means, or can be computed additionally in $O(d m n)$ time. The training process on a dataset of $70 \mathrm{~K}$ items with 784 dimensions can thus be achieved in a few seconds on a standard desktop PC.

Connection to the Nyström method As Equation (4), the Nyström eigenfunction by Bengio et al. [2] also generalizes to a new point by a linear combination of a set of low dimensional embeddings:

$$
\phi(\mathbf{x})=\sqrt{n} \sum_{j=1}^{n} \tilde{\mathrm{K}}\left(\mathbf{x}, \mathbf{x}_{j}\right) \mathbf{V}_{r}^{j} \boldsymbol{\Sigma}_{r}^{-1} .
$$

For LE, $\mathbf{V}_{r}$ and $\boldsymbol{\Sigma}_{r}$ correspond to the top $r$ eigenvectors and eigenvalues of a normalized kernel matrix $\tilde{K}$ with

$$
\tilde{K}_{i j}=\tilde{k}\left(\mathbf{x}_{i}, \mathbf{x}_{j}\right)=\frac{1}{n} \frac{\mathrm{w}\left(\mathbf{x}_{i}, \mathbf{x}_{j}\right)}{\sqrt{E_{\mathbf{x}}\left[\mathrm{w}\left(\mathbf{x}_{i}, \mathbf{x}\right)\right] E_{\mathbf{x}}\left[\mathrm{w}\left(\mathbf{x}, \mathbf{x}_{j}\right)\right]}} .
$$

In AGH [31], the formulated hash function was proved to be the corresponding Nyström eigenfunction with the approximate low-rank affinity matrix. The Laplacian eigenmaps latent variable model (LELVM) [5] also formulated the outof-sample mappings for LE in a manner similar to (4) by combining latent variable models. Both of these methods, and the proposed one, can thus be seen as applications of the Nyström method. Note, however, that the suggested method differs in that it is not restricted to spectral methods such as LE, and that the present study aims to learn binary hash functions for similarity-based search rather than dimensionality reduction. LELVM [5] cannot be applied to other embedding methods other than LE.

\section{Stochastic neighborhood preserving hashing}

In order to demonstrate the proposed approach, a hashing method based on t-SNE [41], a non-spectral embedding method, is derived below. T-SNE is a modification of stochastic neighborhood embedding (SNE) [19] which aims to overcome the tendency of that method to crowd points together in one location. t-SNE provides an effective technique for visualizing data and dimensionality reduction, which is capable of preserving local structures in the high dimensional data while retaining some global structures [41]. These properties make t-SNE a good choice for nearest neighbor search. Moreover, as stated in [42], the cost function of t-SNE in fact maximizes the smoothed recall [42] of query points and their neighbors.

The original t-SNE does not scale well, as it has a time complexity which is quadratic in $n$. More significantly, however, it has a non-parametric form, which means that there is no simple function which may be applied to out-of-sample data in order to calculate their coordinates in the embedded space. As was proposed in the previous subsection, one first applies t-SNE [41] to the base set $\mathbf{B}$,

$$
\min _{\mathbf{Y}_{\mathbf{B}}} \sum_{\mathbf{x}_{i} \in \mathbf{B}} \sum_{\mathbf{x}_{j} \in \mathbf{B}} p_{i j} \log \left(\frac{p_{i j}}{q_{i j}}\right) .
$$

Here $p_{i j}$ is the symmetrized conditional probability in the high dimensional space, and $q_{i j}$ is the joint probability defined using the t-distribution in the low dimensional embedding space. The optimization problem (11) is easily solved by a gradient descent procedure 1 After obtaining the embeddings $\mathbf{Y}_{\mathbf{B}}$ of samples $\mathbf{x}_{i} \in \mathbf{B}$, the hash codes for the entire dataset can be easily computed using 9 . This method is labelled IMH-tSNE.

\section{E. Hashing with relaxed similarity preservation}

As in the last subsection, one can compute $\mathbf{Y}_{\mathbf{B}}$ considering local smoothness only within $\mathbf{B}$. Based on equation (4), in this subsection, $\mathbf{Y}_{\mathbf{B}}$ is alternatively computed by considering the

\footnotetext{
${ }^{1}$ See details in [41]. A Matlab implementation of t-SNE is provided by the
} authors of [41] at http://homepage.tudelft.nl/19j49/t-SNE.html. 


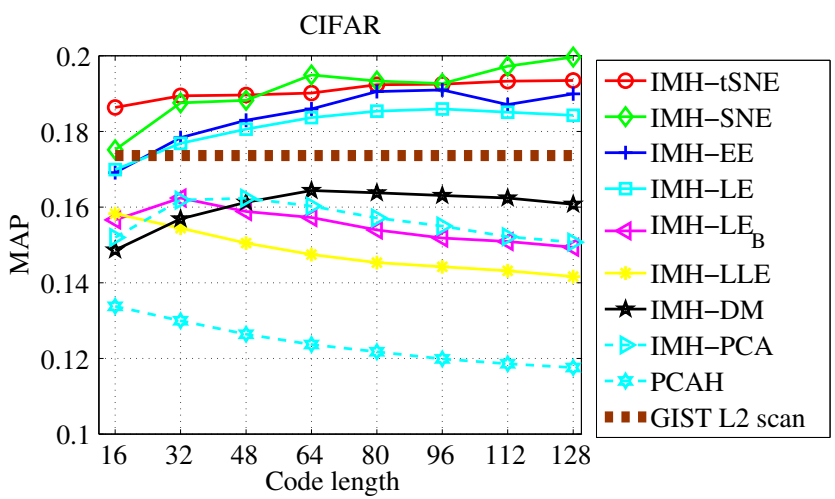

Fig. 3: Comparison among different manifold learning methods within the proposed IMH hashing framework on CIFAR10. IMH with the linear PCA (IMH-PCA) and PCAH [43] are also evaluated for comparison. For clarity, for IMH-LE in Section III-E IMH with the original LE algorithm on the

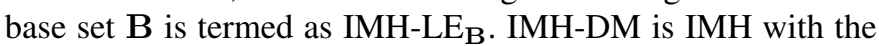
diffusion maps of [26]. The base set size is set to 400 .

smoothness both within $\mathbf{B}$ and between $\mathbf{B}$ and $\mathbf{X}$. As in [8], the objective can be easily obtained by modifying (1) as:

$$
\begin{aligned}
\mathcal{C}\left(\mathbf{Y}_{\mathbf{B}}\right) & =\sum_{\mathbf{x}_{i}, \mathbf{x}_{j} \in \mathbf{B}} \mathrm{w}\left(\mathbf{x}_{i}, \mathbf{x}_{j}\right)\left\|\mathbf{y}_{i}-\mathbf{y}_{j}\right\|^{2} \quad\left(\mathcal{C}_{\mathbf{B B}}\right) \\
& +\lambda \sum_{\mathbf{x}_{i} \in \mathbf{B}, \mathbf{x}_{j} \in \mathbf{X}} \mathrm{w}\left(\mathbf{x}_{i}, \mathbf{x}_{j}\right)\left\|\mathbf{y}_{i}-\mathbf{y}_{j}\right\|^{2} \quad\left(\mathcal{C}_{\mathbf{B X}}\right)
\end{aligned}
$$

where $\lambda$ is the trade-off parameter. $\mathcal{C}_{\mathrm{BB}}$ enforces smoothness of the learned embeddings within $\mathbf{B}$ while $\mathcal{C}_{\mathbf{B X}}$ ensures the smoothness between $\mathbf{B}$ and $\mathbf{X}$. This formulation is actually a relaxation of (1), by discarding the part which minimizes the dissimilarity within $\mathbf{X}$ (denoted as $\mathcal{C}_{\mathbf{X X}}$ ). $\mathcal{C}_{\mathbf{X X}}$ is ignored since computing the similarity matrix within $\mathbf{X}$ costs $O\left(n^{2}\right)$ time. The smoothness between points in $\mathbf{X}$ is implicitly ensured by (10).

Applying equation (10) for $\mathbf{y}_{j}, j \in \mathbf{X}$ to (12), one obtains the following problem

$$
\begin{aligned}
& \min \operatorname{trace}\left(\mathbf{Y}_{\mathbf{B}}^{\top}\left(\mathbf{D}_{\mathbf{B}}-\mathbf{W}_{\mathbf{B}}\right) \mathbf{Y}_{\mathbf{B}}\right) \\
& \quad+\lambda \operatorname{trace}\left(\mathbf{Y}_{\mathbf{B}}^{\top}\left(\mathbf{D}_{\mathbf{B X}}-\overline{\mathbf{W}}_{\mathbf{X B}}^{\top} \mathbf{W}_{\mathbf{X B}}\right) \mathbf{Y}_{\mathbf{B}}\right),
\end{aligned}
$$

where $\mathbf{D}_{\mathbf{B}}=\operatorname{diag}\left(\mathbf{W}_{\mathbf{B}} \mathbf{1}\right)$ and $\mathbf{D}_{\mathbf{B X}}=\operatorname{diag}\left(\mathbf{W}_{\mathbf{B X}} \mathbf{1}\right)$ are both $m \times m$ diagonal matrices. Taking the constraint in (1), one obtains

$$
\begin{aligned}
& \min _{\mathbf{Y}_{\mathbf{B}}} \operatorname{trace}\left(\mathbf{Y}_{\mathbf{B}}^{\top}(\mathbf{M}+\lambda \mathbf{T}) \mathbf{Y}_{\mathbf{B}}\right) \\
& \text { s.t. } \mathbf{Y}_{\mathbf{B}}^{\top} \mathbf{Y}_{\mathbf{B}}=m \mathbf{I}
\end{aligned}
$$

where $\mathbf{M}=\mathbf{D}_{\mathbf{B}}-\mathbf{W}_{\mathbf{B}}, \mathbf{T}=\mathbf{D}_{\mathbf{B X}}-\overline{\mathbf{W}}_{\mathbf{X B}}^{\top} \mathbf{W}_{\mathbf{X B}}$. The optimal solution $\mathbf{Y}_{\mathbf{B}}$ of the above problem is easily obtained by identifying the $r$ eigenvectors of $\mathbf{M}+\lambda \mathbf{T}$ corresponding to the smallest eigenvalues (excluding the eigenvalue 0 with respect to the trivial eigenvector 1$)^{2}$. This method is named IMH-LE in the following text.

\footnotetext{
${ }^{2}$ The parameter $\lambda$ is set to 2 in all experiments.
}

\section{F. Manifold learning methods for hashing}

In this section, different manifold learning methods are compared for hashing within the proposed IMH framework. Figure 3 reports the comparative results in mean of average precision (MAP). For comparison, the linear PCA is also evaluated within the framework (IMH-PCA in the figure). As can be clearly seen that, IMH-tSNE, IMH-SNE and IMH-EE perform slightly better than IMH-LE (Section III-E). This is mainly because these three methods are able to preserve local neighborhood structure while, to some extent, preventing data points from crowding together. It is promising that all of these methods perform better than an exhaustive $\ell_{2}$ scan using the uncompressed GIST features.

Figure 3 shows that LE (IMH-LE ${ }_{\mathbf{B}}$ in the figure), the most widely used embedding method in hashing, does not perform as well as a variety of other methods (e.g., t-SNE), and in fact performs worse than PCA, which is a linear technique. This is not surprising because LE (and similarly LLE) tends to collapse large portions of the data (and not only nearby samples in the original space) close together in the lowdimensional space. The results are consistent with the analysis in [41], [4]. Based on the above observations, we argue that manifold learning methods (e.g. t-SNE, EE), which not only preserve local similarity but also force dissimilar data apart in the low-dimensional space, are more effective than the popular LE for hashing. Preserving the global structures of the data is critical for the manifold learning algorithms like t-SNE used in the proposed IMH framework. The previous work called spline regression hashing (SRH) [32] also exploited both the local and global data similarity structures of data via a Laplacian matrix, which can decrease over-fitting, as discussed in [32]. SRH captures the global similarity structure of data through constructing global non-linear hash functions, while, in the proposed method, capturing the global structures (i.e., by $\mathrm{t}-$ SNE) is only applied on the small base set and the hash function is formulated by a locally linear regression model.

It is interesting to see that IMH-PCA outperforms PCAH [43] by a large margin, despite the fact that PCA is performed on the whole training data set by PCAH. This shows that the generalization capability of IMH based on a very small set of data points also works for linear dimensionality methods.

\section{EXPERIMENTAL RESULTS}

IMH is evaluated on four large scale image datasets: CIFAR-10 3 , MNIST , SIFT1M [43] and GIST1M 4 ] The MNIST dataset consists of 70,000 images, each of 784 dimensions, of handwritten digits from ' 0 ' to ' 9 '. As a subset of the well-known $80 \mathrm{M}$ tiny image collection [40], CIFAR10 consists of 60,000 images which are manually labelled as 10 classes with 6,000 samples for each class. Each image in this dataset is represented by a GIST feature vector [34] of dimension 512. For MNIST and CIFAR-10, the whole dataset is split into a test set with 1,000 samples and a training set with all remaining samples.

$\sqrt[3]{\text { http://www.cs.toronto.edu/ kriz/cifar.html }}$

4 http://corpus-texmex.irisa.fr/ 

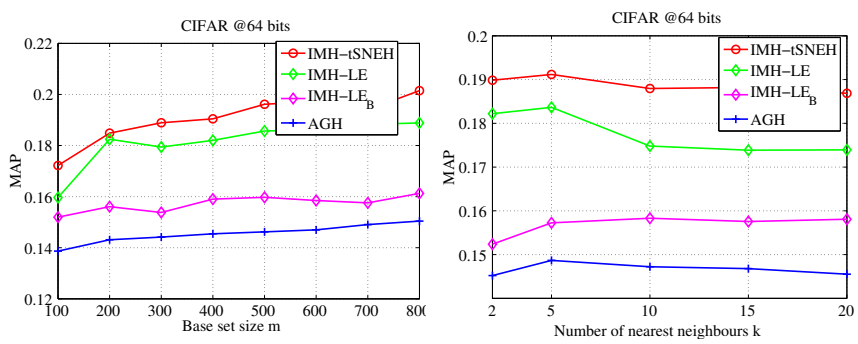

Fig. 4: MAP results versus varying base set size $m$ (left, fixing $k=5$ ) and number of nearest base points $k$ (right, fixing $m=$ 400) for the proposed methods and AGH. The comparison is conducted on the CIFAR-10 dataset using 64-bits .

TABLE I: MAP (\%) of different base generating methods: random sampling vs. K-means. The comparison is performed on the CIFAR-10 dataset with code lengths from 32 to 96 and base set size 400. Average results (standard deviation) are given based on 10 runs.

\begin{tabular}{cc|cccc}
\hline \hline Bits & Method & IMH-LE $_{\mathbf{B}}$ & IMH-LE & IMH-tSNE & AGH \\
\hline \multirow{3}{*}{32} & Random & $14.07(0.27)$ & $16.20(0.19)$ & $17.26(0.43)$ & - \\
& K-medians & $15.14(0.15)$ & $17.08(0.21)$ & $17.32(0.27)$ & - \\
& K-means & $16.05(0.16)$ & $17.48(0.44)$ & $\mathbf{1 8 . 3 8}(\mathbf{0 . 3 6})$ & $15.76(0.17)$ \\
\hline \multirow{3}{*}{64} & Random & $14.64(0.17)$ & $16.98(0.21)$ & $16.93(0.49)$ & - \\
& K-medians & $15.43(0.12)$ & $17.11(0.17)$ & $17.62(0.31)$ & - \\
& K-means & $15.90(0.11)$ & $18.20(0.37)$ & $\mathbf{1 9 . 0 4 ( 0 . 2 7 )}$ & $14.55(0.13)$ \\
\hline \multirow{2}{*}{96} & Random & $14.76(0.20)$ & $17.02(0.23)$ & $17.21(0.48)$ & - \\
& K-medians & $15.42(0.19)$ & $17.21(0.28)$ & $17.73(0.28)$ & - \\
& K-means & $15.46(0.09)$ & $18.56(0.34)$ & $\mathbf{1 9 . 4 1 ( \mathbf { 0 . 2 3 } )}$ & $13.98(0.10)$ \\
\hline \hline
\end{tabular}

Nine hashing algorithms is compared, including the proposed IMH-tSNE, IMH-LE and seven other unsupervised state-of-the-art methods: PCAH [43], SH [46], AGH [31] and STH [48], BRE [23], ITQ [13], Spherical Hashing ( $\mathrm{SpH})$ [17]. The provided codes and suggested parameters according to the authors of these methods are used. Due to the high computational cost of BRE and high memory cost of STH, 1,000 and 5,000 training points are sampled for these two methods respectively. The performance is measured by MAP or precision and recall curves for Hamming ranking using 16 to 128 hash bits. The results for hash lookup using a Hamming radius within 2 by $\mathrm{F} 1$ score [33]: $F_{1}=$ $2($ precision $\cdot$ recall $) /($ precision + recall $)$ are also reported. Ground truths are defined by the category information for the labeled datasets MNIST and CIFAR-10, and by Euclidean neighbors for SIFT1M and GIST1M.

\section{A. Base selection}

In this section, the CIFAR-10 dataset is taken as an example to compare different base generation methods and different base sizes for the proposed methods. AGH is also evaluated here for comparison. Table II compares three methods for generating base point sets: random sampling, K-medians and $\mathrm{K}$-means on the training data. One can easily see that the performance of the proposed methods using K-means is better at all code lengths than that using other two methods. It is also clear from Table $[$ that the K-medians algorithm achieves better performance than random sampling, however still inferior to K-means.
Different from K-means that constructs each base point by averaging the data points in the corresponding cluster, random sampling and K-medians generate the base set with real data samples in the training set. However this property does not help K-medians obtain better hash codes than K-means. This is possibly because K-means produces the cluster centers with minimum quantization distortions at each group of the data points. Another advantage of K-means is it is much more efficient than K-medians.

As can also be seen, even with base set by random sampling, the proposed methods outperform AGH in all cases but one. Due to the superior results and high efficiency in practice, the base set is generated by K-means in the following experiments.

From Figure 4, it is clear that the performance of the proposed methods is consistently improved with the increasing base set size $m$, which is consistent with the analysis of the prototype algorithm. One can observe that, the performance does not change significantly with the number of nearest base points $k$. It is also clear that IMH-LE $\mathrm{B}_{\mathbf{B}}$, which only enforces smoothness in the base set, does not perform as well as IMHLE, which also enforces smoothness between the base set and training set.

To further investigate the impact of the base set size $m$, in both performance and efficiency, the proposed methods are performed with large variance of $m$ on CIFAR-10 and MNIST. The results for the proposed IMH-tSNE are shown in Table II As can be clearly seen, for the dataset of CIFAR-10, the MAP score by IMH-tSNE improves consistently with the increasing base set size $m$ when $m \leq 400$, while does not change dramatically with larger $m$. On MNIST, the performance of IMH-tSNE is also consistently improved when $m$ increases. Same as on CIFAR-10, the MAP score does not significantly change with larger $m$.

In terms of computational efficiency, on both datasets, the training time cost is considerably increased with larger $m$. For example, IMH-tSNE costs about 4.3 seconds with 400 base samples, while costs more than 21 seconds with 1,000 base samples on the CIFAR-10 dataset. For the retrieval task, the testing time is more crucial. As shown in Table II the testing time is in general linearly increased with $m$. With a small $m$, the testing is very efficient. When with a large $m$ (e.g., $m \geq 3000$ ), IMH-tSNE needs more than one milliseconds to compute the binary codes of the query, which is not scalable for large-scale tasks. Take both performance and computational efficiency into account, for the remainder of this paper, the settings $m=400$ and $k=5$ are used for the proposed methods, unless otherwise specified.

Figure 5 shows the t-SNE embeddings of the base set (400 points generated by K-means) and the embeddings of the whole MNIST dataset computed by the proposed inductive method (without binarization). As can be seen from the right figure that most of the points are close to their corresponding clusters (with respect to 10 digits). This observation shows that, in the low-dimensional embedding space, the proposed method can well preserve the local manifold structure of the whole dataset based on a relatively small base set. 
TABLE II: Impact of the base set size $m$ of IMH-tSNE on the retrieval performance and computational efficiency. The base set is generated by K-means. The results with 64 bits are reported, based on 10 independent runs. The experiments are conducted on a desktop PC with a 4-core 3.40GHZ CPU and 32G RAM.

\begin{tabular}{|c|c|c|c|c|c|c|c|c|c|c|c|c|}
\hline & $m$ & 100 & 200 & 400 & 800 & 1000 & 1500 & 2000 & 3000 & 4000 & 5000 & 10000 \\
\hline \multirow{3}{*}{ CIFAR } & MAP (\%) & 17.55 & 18.19 & 19.52 & 19.99 & 20.11 & 20.13 & 20.05 & 20.23 & 20.37 & 20.45 & 20.85 \\
\hline & Training Time (s) & 0.7754 & 1.3913 & 4.2850 & 13.5576 & 21.3487 & 53.5668 & 97.6969 & 229.6100 & 419.4027 & 664.0867 & 2701.8001 \\
\hline & Testing Time (ms) & 0.0057 & 0.0095 & 0.0157 & 0.0254 & 0.0308 & 0.0475 & 0.0621 & 0.1045 & 0.1388 & 0.1981 & 0.3634 \\
\hline \multirow{3}{*}{ MNIST } & MAP (\%) & 57.01 & 67.72 & 77.78 & 79.71 & 80.40 & 82.52 & 82.10 & 86.47 & 85.66 & 87.37 & 87.74 \\
\hline & Training Time (s) & 0.9546 & 1.6804 & 4.6302 & 814.7084 & 22.7297 & 54.7796 & 102.1419 & 232.8753 & 423.6395 & 669.6443 & 2707.8012 \\
\hline & Testing Time (ms) & 0.0074 & 0.0122 & 0.0182 & 0.0330 & 0.0394 & 0.0579 & 0.0984 & 0.1222 & 0.2032 & 0.2293 & 0.4357 \\
\hline
\end{tabular}

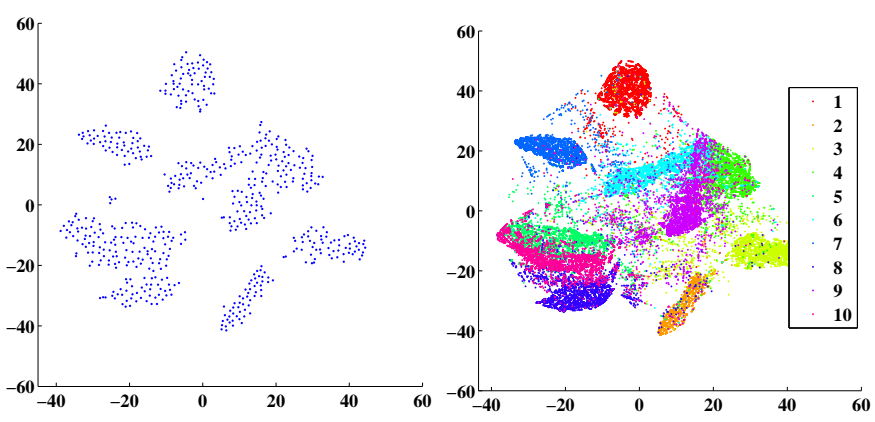

Fig. 5: Visualization of the digits in MNIST in 2D by $t-$ SNE: (left) t-SNE embeddings of the base set (400 data points generated by K-means); (right) embeddings of all the 60,000 samples computed by the proposed inductive method. In the right, the labels of the digits are indicated with 10 different colors.

\section{B. Results on CIFAR-10 dataset}

The comparative results based on MAP for Hamming ranking with code lengths from 16 to 128 bits are reported in Figure 6. It can be seen that the proposed IMH-LE and IMH-tSNE perform best in all cases. Among the proposed algorithms, the LE based IMH-LE is inferior to the t-SNE based IMH-tSNE. IMH-LE is still much better than AGH and STH, however. ITQ performs better than SpH and BRE on this dataset, but is still inferior to IMH. SH and PCAH perform worst in this case. This is because $\mathrm{SH}$ relies upon its uniform data assumption while PCAH simply generates the hash hyperplanes by PCA directions, which does not explicitly capture the similarity information. The results are consistent with the complete precision and recall curves shown in the supplementary material. The $F_{1}$ results for hash lookup with Hamming radius 2 are also reported. It is can be seen that IMH-LE and IMH-tSNE also outperform all other methods by large margins. BRE and AGH obtain better results than the remaining methods, although the performance of all methods drop as code length grows.

Figure 7 shows the precision and recall curves of Hamming ranking for the compared methods. STH and AGH obtain relatively high precisions when a small number of samples are returned, however precision drops significantly as the number of retrieved samples increases. In contrast, IMH-tSNE, IMHLE and ITQ achieve higher precisions with relatively larger numbers of retrieved points.

Figure 8 shows the qualitative results of IMH and related methods on some sample queries. As can be seen, IMH-tSNE
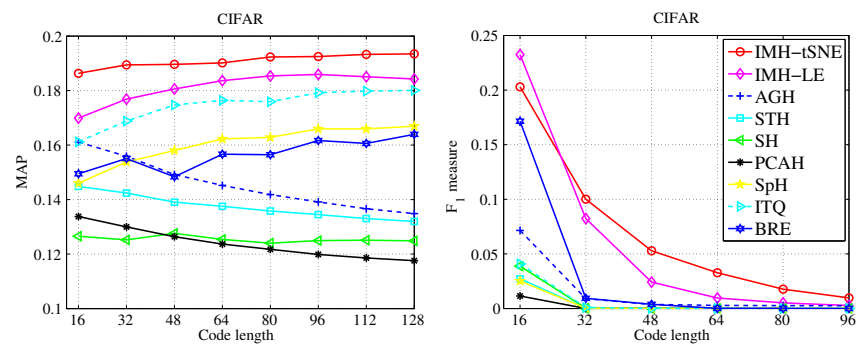

Fig. 6: Comparison of different methods on CIFAR-10 based on MAP (left) and $F_{1}$ (right) for varying code lengths.
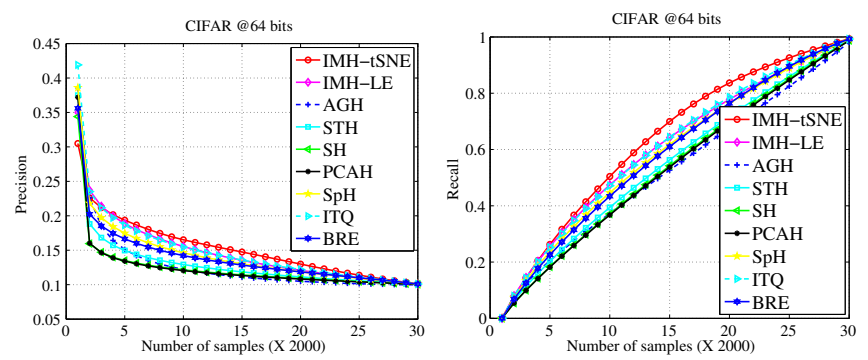

Fig. 7: Comparison of different methods on CIFAR-10 based on precision (left) and recall (right) using 64-bits. Please refer to the complementary for complete results for other code lengths.

achieves the best search quality in term of visual relevance.

\section{Results on MNIST dataset}

The MAP and $F_{1}$ scores for these compared methods are reported in Figure 13. As in Figure 6, IMH-tSNE achieves the best results. It is clear that, on this dataset IMH-tSNE outperforms IMH-LE by a large margin, which increases as code length increases. This further demonstrates the advantage of t-SNE as a tool for hashing by embedding high dimensional data into a low dimensional space. The dimensionality reduction procedure not only preserves the local neighborhood structure, but also reveals important global structure (such as clusters) [41]. Among the four LE-based methods, while IMHLE shows a small advantage over AGH, both methods achieve much better results than STH and SH. ITQ and BRE obtain high MAPs with longer bit lengths, but they still perform less well for the hash look up $F_{1}$. PCAH performs worst in terms of both MAP and the $F_{1}$ measure. Refer to the supplementary material for the complete precision and recall curves which validate the observations here. 


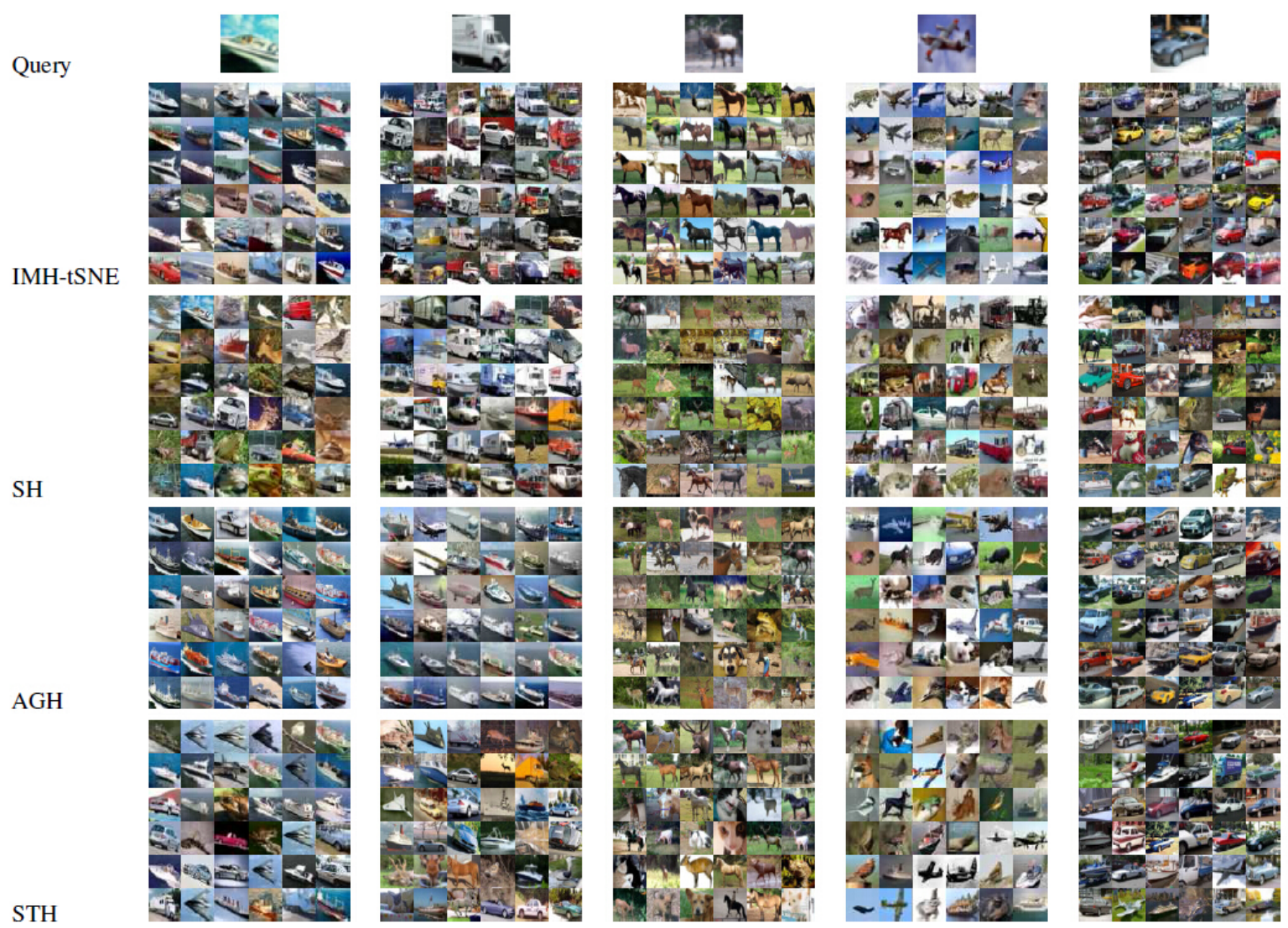

Fig. 8: The query images (top row) and the retrieved images by various methods with 32 hash bits.
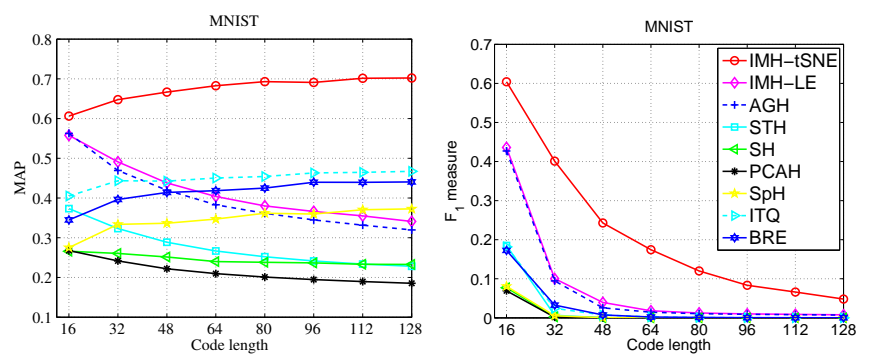

Fig. 9: Comparison of different methods on the MNIST dataset using MAP (left) and $F_{1}$ (right) for varying code lengths.

Efficiency Table III shows training and testing time on the MNIST dataset for various methods, and shows that the linear method, PCAH, is fastest. IMH-tSNE is slower than IMH$\mathrm{LE}, \mathrm{AGH}$ and $\mathrm{SH}$ in terms of training time, however all of these methods have relatively low execution times and are much faster than STH and BRE. In terms of test time, both IMH algorithms are comparable to other methods, except STH which takes much more time to predict the binary codes by SVM on this non-sparse dataset.
TABLE III: Comparison of training and testing times (in seconds) on MNIST with 70K 784D feature points. K-means dominates the cost of AGH and IMH (8.9 seconds), which can be conducted in advance in practice. The experiments are based on a desktop PC with a 4-core 3.07GHZ CPU and $8 \mathrm{G}$ RAM.

\begin{tabular}{ccc|cc}
\hline \hline Method & \multicolumn{2}{c|}{ Train time } & \multicolumn{2}{c}{ Test time } \\
& 64-bits & 128-bits & 64-bits & 128 -bits \\
\hline IMH-LE & 9.9 & 9.9 & $5.1 \times 10^{-5}$ & $3.8 \times 10^{-5}$ \\
IMH-tSNE & 16.7 & 20.2 & $2.8 \times 10^{-5}$ & $3.1 \times 10^{-5}$ \\
SH & 6.8 & 16.2 & $5.8 \times 10^{-5}$ & $1.8 \times 10^{-4}$ \\
STH & 266.1 & 485.4 & $1.8 \times 10^{-3}$ & $3.6 \times 10^{-3}$ \\
AGH & 9.5 & 9.5 & $4.7 \times 10^{-5}$ & $5.5 \times 10^{-5}$ \\
PCAH & 3.8 & 4.1 & $5.7 \times 10^{-6}$ & $1.2 \times 10^{-5}$ \\
SpH & 19.7 & 41.0 & $1.3 \times 10^{-5}$ & $2.0 \times 10^{-5}$ \\
ITQ & 10.4 & 20.3 & $6.9 \times 10^{-6}$ & $1.1 \times 10^{-5}$ \\
BRE & 418.9 & 1731.9 & $1.2 \times 10^{-5}$ & $2.4 \times 10^{-5}$ \\
\hline \hline
\end{tabular}

\section{Results on SIFT1M and GISTIM}

SIFT1M contains one million local SIFT descriptors extracted from a large set of images [43], each of which is represented by a $128 \mathrm{D}$ vector of histograms of gradient orientations. GIST1M contains one million GIST features and each feature is represented by a $960 \mathrm{D}$ vector. For both of 

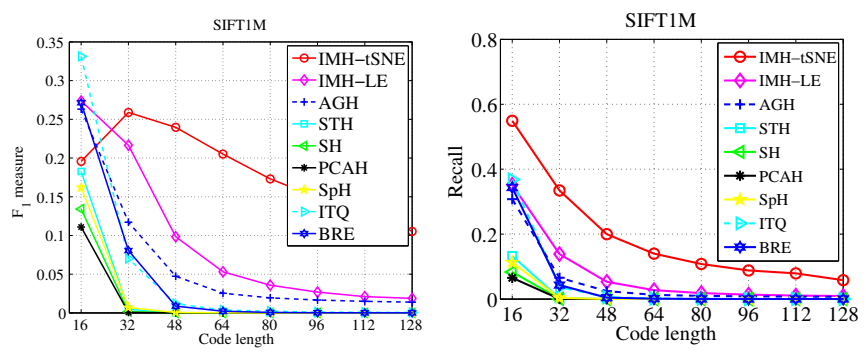

Fig. 10: Comparative results results on SIFT1M for $F_{1}$ (left) and recall (right) with Hamming radius 2. Ground truth is defined to be the closest 2 percent of points as measured by the Euclidean distance.
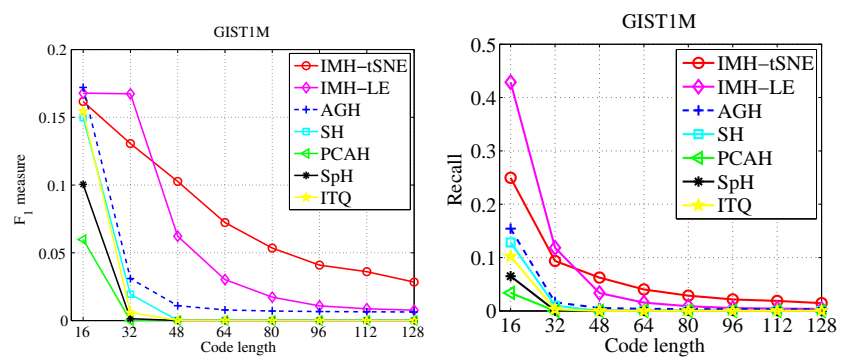

Fig. 11: Comparative results results on GIST1M by $F_{1}$ (left) and recall (right) with Hamming radius 2. Ground truth is defined to be the closest 2 percent of points as measured by the Euclidean distance.

these datasets, one million samples are used as training set and additional $10 \mathrm{~K}$ are used for testing. As in [43], ground truth is defined as the closest 2 percent of points as measured by the Euclidean distance. For these two large datasets, 1, 000 points are generated by K-means and $k$ is set as 2 for both IMH and AGH. The comparative results on SIFT1M and GIST1M are summarized in Figure 10 and Figure 11, respectively. Again, IMH consistently achieves superior results in terms of both $F_{1}$ score and recall with Hamming radius 2 . As can be seen that, the performance of most of these methods decreases dramatically with increasing code length as the Hamming spaces become more sparse, which makes the hash lookup fail more often. However IMH-tSNE still achieves relatively high scores with large code lengths. If one looks at Figure 10 (left), ITQ obtains the highest $F_{1}$ with 16-bits, however it decreases to near zero at 64-bits. In contrast, IMH-tSNE still manages an $F_{1}$ of 0.2 . Similar results are observed in the recall curves.

\section{E. Classification on binary codes}

In order to demonstrate classification performance a linear SVM is trained on the binary codes generate by IMH for the MNIST data set. In order to learn codes with higher bit lengths for IMH and $\mathrm{AGH}$, the base set size is set to 1,000. Accuracies of different binary encodings are shown in Figure 12. Both IMH and AGH achieve high accuracies on this dataset, although IMH performs better with higher code lengths. In contrast, the best results of all other methods, obtained by ITQ, are consistently worse than those for IMH, especially for short code lengths. Note that even with only 128 -

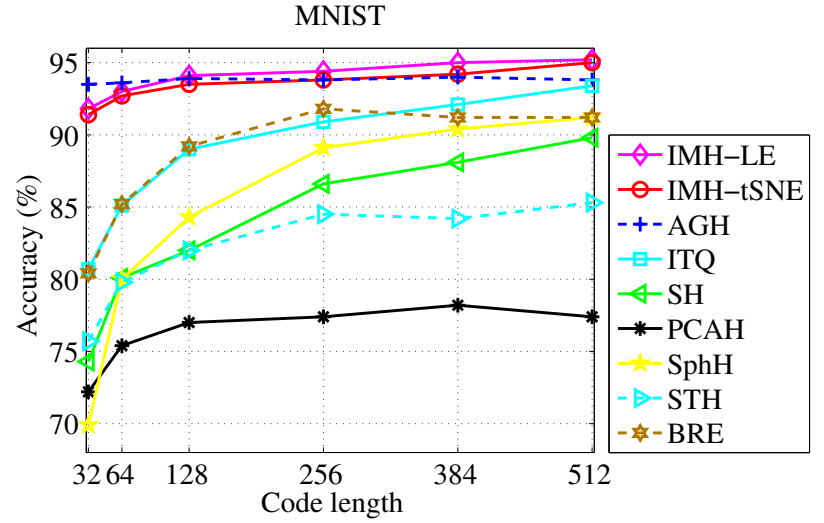

Fig. 12: Classification accuracy (\%) on MNIST with binary codes of various hashing methods by linear SVM.

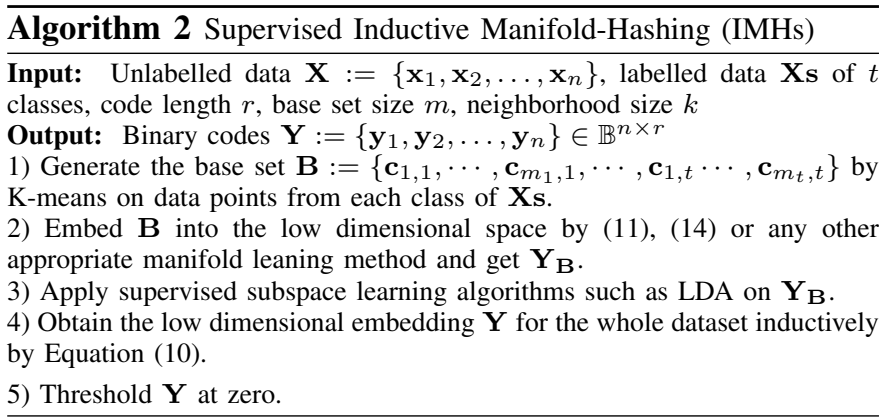

bit binary features IMH obtains a high $94.1 \%$. Interestingly, the same classification rate of $94.1 \%$ is obtained by applying the linear SVM to the uncompressed 784D features, which occupy several hundreds times as much space as the learned hash codes.

\section{MINIMIZE THE QUANTIZATION DISTORTION BY LEARNED ROTATIONS}

In the above sections, the binary codes are obtained by directly thresholding the learned embeddings at zeros. The simple binarization may cause large quantization loss. In this section, learned rotations are applied to minimize the quantization errors. That is, normalize the data points such that they are zero-centred in the embedded space and then orthogonally rotate the normalized embeddings for binarization. Orthogonal rotation has been adopted by many methods (e.g. [21], [13], [15], [9]) to minimize the quantization error. The simple algorithm in ITQ [13] is used for the proposed method.

The impact of the rotations applied on the learned embeddings is evaluated on MNIST and CIFAR. Figure 13 clearly shows that the orthogonal rotations achieve significantly performance improvements (in terms of both $F_{1}$ and recall) for IMH-LE and IMH-tSNE. In conjunctions with the rotations, the proposed IMH-LE and IMH-tSNE methods perform much better than the PCA based PCA-ITQ. Again this result demonstrates the advantages of the proposed manifold hashing method. 

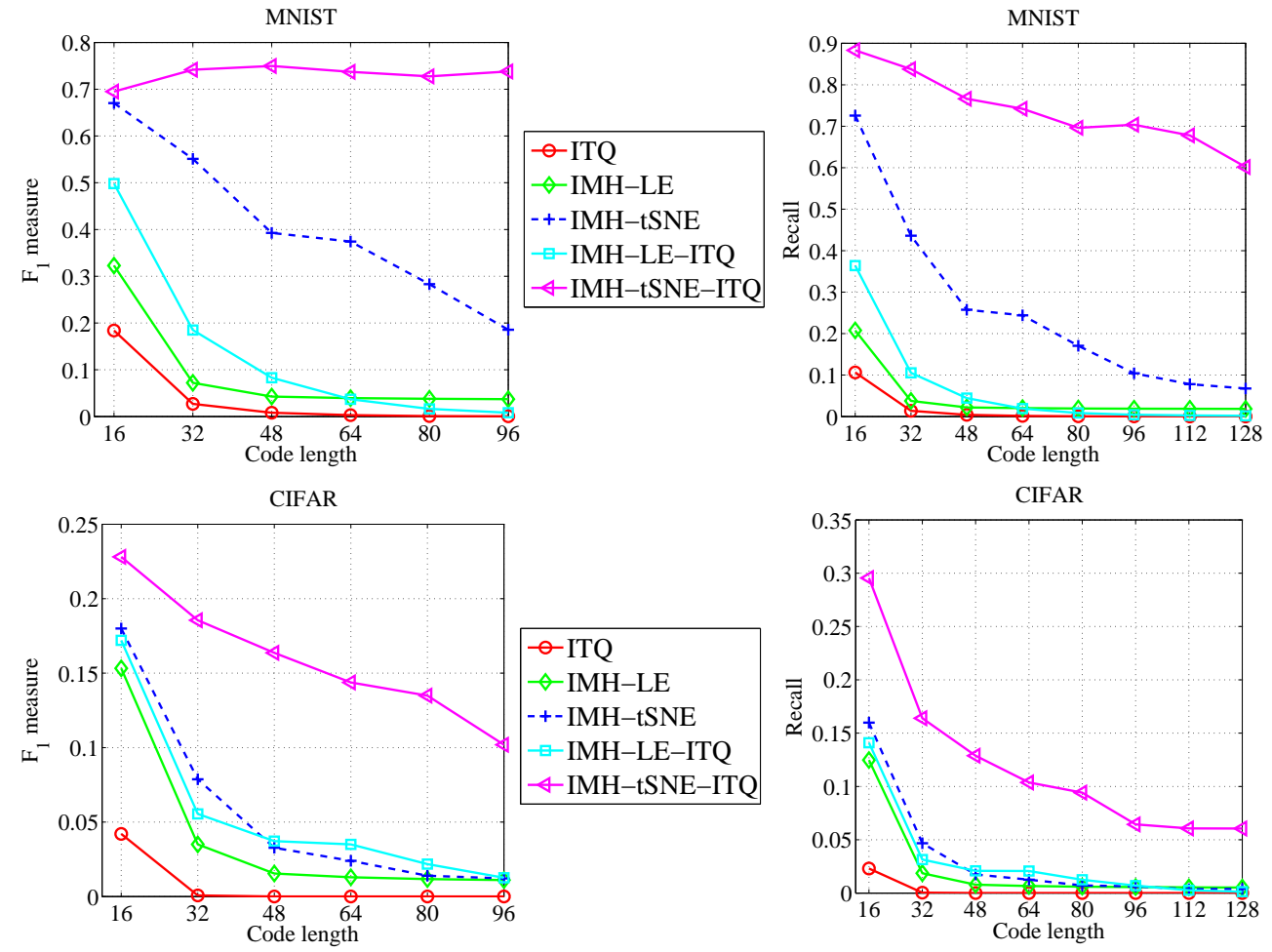

Fig. 13: Evaluation of IMH with ITQ rotations on the MNIST and CIFAR dataset using $F_{1}$ and recall with Hamming radius 2 for varying code lengths.
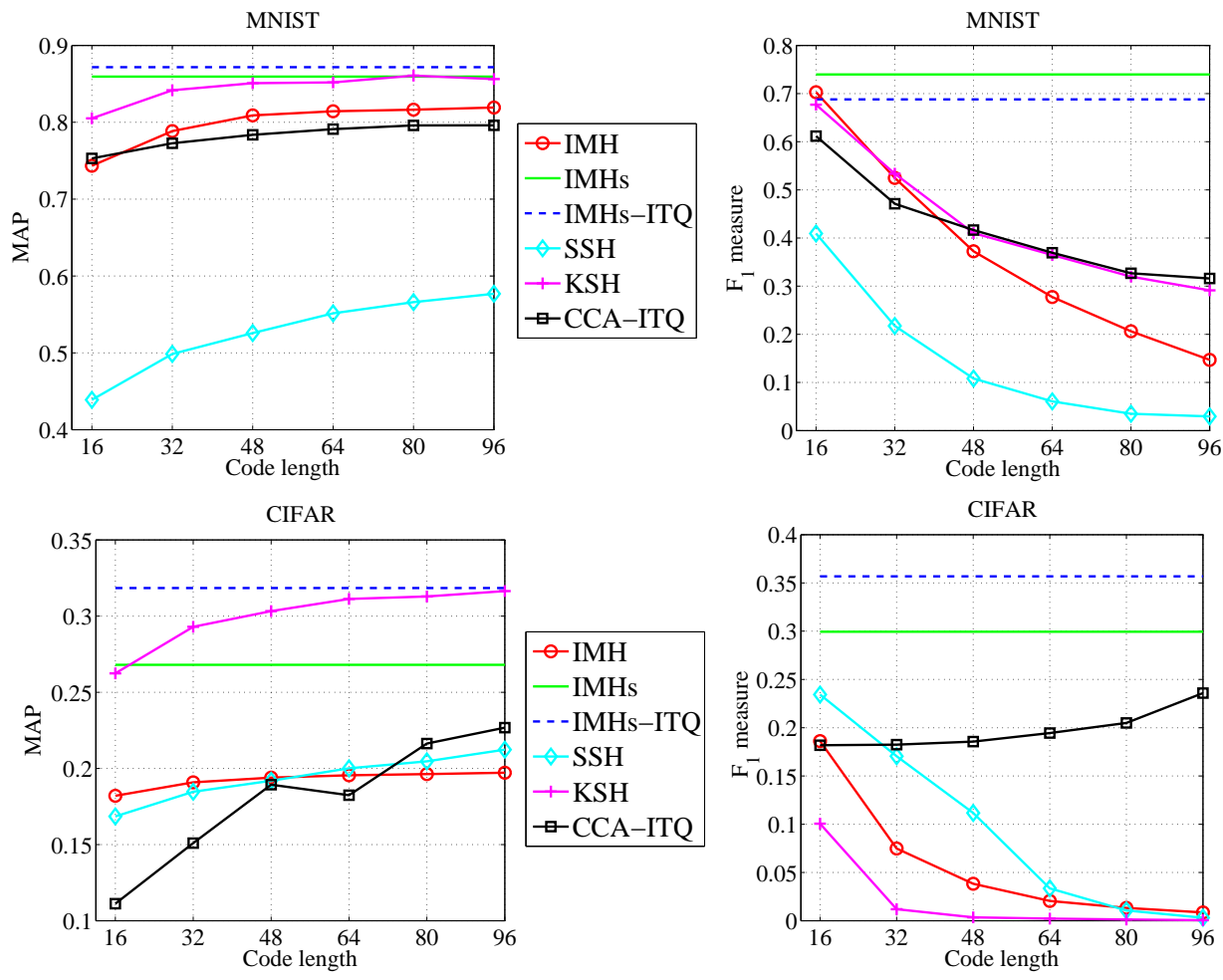

Fig. 14: Evaluation of IMH with supervised learning by LDA on the MNIST and CIFAR datasets. Non-linear embeddings of IMH are obtained by t-SNE. Since there are only 10 classes with both these two datasets, the reduced dimensionality by LDA (thereby the binary code lenght) is set to 9 . 


\section{SEMANTIC HASHING WITH SUPERVISED MANIFOLD} LEARNING

The proposed inductive manifold hashing algorithm has shown to work well on preserving the semantic neighborhood relationships without using label information. It is natural that the performance could be improved by applying supervised learning methods instead of the unsupervised ones to learn the nonlinear embeddings. Unfortunately, most of the manifold learning algorithms are unsupervised. In order to use the large amount of unsupervised manifold learning methods, a straightforward supervised extension to the proposed IMH algorithm is proposed int this study. First, the base set $\mathbf{B}:=\left\{\mathbf{c}_{1,1}, \cdots, \mathbf{c}_{m_{1}, 1}, \cdots, \mathbf{c}_{1, t} \cdots, \mathbf{c}_{m_{t}, t}\right\}$ is generated by applying K-means on data from each of the $t$ classes. After the nonlinear embeddings $\mathbf{Y}_{\mathbf{B}}$ of $\mathbf{B}$ are obtained, the supervised subspace learning algorithms are simply conducted on $\mathbf{Y}_{\mathbf{B}}$. For a new data point, its binary codes are then obtained by 98. The supervised manifold hashing method is summarised in Algorithm 2

In this section, the linear discriminant analysis (LDA) is taken as an example to verify the efficacy of the proposed supervised manifold hashing algorithm. The proposed method is also compared with several recently proposed supervised hashing approaches such as semi-supervised hashing ( $\mathrm{SSH}$ [43]) with sequential projection learning, kernel-based supervised hashing (KSH [30]) and ITQ with Canonical Correlation Analysis (CCA-ITQ [14]).

Experiments are performed on MNIST and CIFAR. In this experiment, 2,000 labelled samples are randomly selected for supervised learning for $\mathrm{SSH}$ and $\mathrm{KSH}$, and 1,000 labelled samples are sampled for the base set for IMHs. All labelled training data are used for the linear CCA-ITQ. Since there are only 10 classes with both these two datasets, the reduced dimensionality by LDA (thereby the binary code length) in the proposed IMHs is fixed to 9 . The results are reported in Figure 14 It is clear that the proposed supervised inductive manifold hashing algorithm IMHs significantly improve the original IMH and other compared supervised methods in both MAP and $F_{1}$ measure. The ITQ rotations (IMHs-ITQ in the figure) further improves IMHs with considerable gains, especially on the CIFAR dataset of natural images. Among other supervised hashing methods, KSH obtains the highest MAPs on MNIST and CIFAR with IMHs or IMHs-ITQ. However it needs much larger binary code lengths to achieve comparable performance with the proposed methods. In terms of $F_{1}$, CCA-ITQ is identified to have the best results with long codes.

From these results, it is clear that label information is very useful to achieve semantically effective hashing codes. And also, the proposed simple supervised hashing framework can effectively leverage the supervised information in the proposed manifold hashing algorithms. Also note that the proposed method does not assume a specific algorithm like LDA, any other supervised subspace learning or metric learning algorithms may further improve the performance.

\section{CONCLUSION AND DISCUSSION}

The present study has proposed a simple yet effective hashing framework, which provides a practical connection between manifold learning methods (typically non-parametric and with high computational cost) and hash function learning (requiring high efficiency). By preserving the underlying manifold structure with several non-parametric dimensionality reduction methods, the proposed hashing methods outperform several state-of-the-art methods in terms of both hash lookup and Hamming ranking on several large-scale retrieval-datasets. The proposed inductive formulation of the hash function sees the suggested methods require only linear time $(O(n))$ for indexing all of the training data and a constant search time for a novel query. Experiments showed that the hash codes can also achieve promising results on a classification problem even with very short code lengths. The proposed inductive manifold hashing method was then extended by applying orthogonal rotations on the learned nonlinear embeddings to minimize the quantization errors, which was shown to achieve significant performance improvements. In addition, this work further extended IMH by adopting supervised subspace learning on the data manifolds, which provided an effective supervised manifold hashing framework.

The proposed hashing methods have been shown to work well on the image retrieval and classification tasks. As an efficient and effective nonlinear feature extraction method, this algorithm can also be applied to other applications, especially the ones need to deal with large dataset. For example, the introduced hashing techniques can be applied to large-scale mobile video retrieval [29]. Another useful application of the hashing methods would be compressing the high dimensional features into short binary codes, which could significantly speed up the potential tasks, such as the large scale ImageNet image classification [12], [28].

In this work, the base set size $m$ was set empirically, which was not an optimal choice apparently. How to automatically set this parameter according to the size and distribution of a specific dataset deserves a future study.

\section{ACKNOWLEDGEMENTS}

This work was in part supported by ARC Future Fellowship FT120100969.

\section{REFERENCES}

[1] M. Belkin and P. Niyogi. Laplacian eigenmaps and spectral techniques for embedding and clustering. In Proc. Adv. Neural Inf. Process. Syst., 2001.

[2] Y. Bengio, O. Delalleau, N. Roux, J. Paiement, P. Vincent, and M. Ouimet. Learning eigenfunctions links spectral embedding and kernel PCA. Neural Comput., 16(10):2197-2219, 2004.

[3] M. M. Bronstein and P. Fua. LDAHash: Improved matching with smaller descriptors. IEEE Trans. Pattern Anal. Mach. Intell., 2012.

[4] M. Carreira-Perpinán. The elastic embedding algorithm for dimensionality reduction. In Proc. Int. Conf. Mach. Learn., 2010.

[5] M. Carreira-Perpinán and Z. Lu. The laplacian eigenmaps latent variable model. Proc. Int. Conf. Artif. Intell. Stat., 2007.

[6] R. Chaudhry and Y. Ivanov. Fast approximate nearest neighbor methods for non-euclidean manifolds with applications to human activity analysis in videos. In Proc. Eur. Conf. Comp. Vis., 2010.

[7] M. Datar, N. Immorlica, P. Indyk, and V. Mirrokni. Locality-sensitive hashing scheme based on p-stable distributions. In Ann. Symp. Comput. Geometry, 2004. 
[8] O. Delalleau, Y. Bengio, and N. Le Roux. Efficient non-parametric function induction in semi-supervised learning. In Proc. Int. Workshop Artif. Intelli. Stat., pages 96-103, 2005.

[9] T. Ge, K. He, Q. Ke, and J. Sun. Optimized product quantization for approximate nearest neighbor search. In Proc. IEEE Conf. Comp. Vis. Patt. Recogn., 2013.

[10] T. Ge, K. He, and J. Sun. Graph cuts for supervised binary coding. In Proc. Eur. Conf. Comp. Vis., 2014.

[11] A. Gionis, P. Indyk, and R. Motwani. Similarity search in high dimensions via hashing. In Proc. Int. Conf. Very Large Datadases, 1999

[12] Y. Gong, S. Kumar, H. A. Rowley, and S. Lazebnik. Learning binary codes for high-dimensional data using bilinear projections. In Proc. IEEE Conf. Comp. Vis. Patt. Recogn., pages 484-491, 2013.

[13] Y. Gong and S. Lazebnik. Iterative quantization: A procrustean approach to learning binary codes. In Proc. IEEE Conf. Comp. Vis. Patt. Recogn., 2011.

[14] Y. Gong, S. Lazebnik, A. Gordo, and F. Perronnin. Iterative quantization: A procrustean approach to learning binary codes for large-scale image retrieval. IEEE Trans. Pattern Anal. Mach. Intell., 35(12):2916-2929, 2013.

[15] K. He, F. Wen, , and J. Sun. K-means hashing: an affinity-preserving quantization method for learning binary compact codes. In Proc. IEEE Conf. Comp. Vis. Patt. Recogn., 2013.

[16] X. He, W.-Y. Ma, and H.-J. Zhang. Learning an image manifold for retrieval. In Proc. ACM Multimedia, 2004.

[17] J. Heo, Y. Lee, J. He, S. Chang, and S. Yoon. Spherical hashing. In Proc. IEEE Conf. Comp. Vis. Patt. Recogn., 2012.

[18] R. Herbrich and R. C. Williamson. Algorithmic luckiness. J. Mach. Learn. Res., 3:175-212, 2002.

[19] G. Hinton and S. Roweis. Stochastic neighbor embedding. In Proc. Adv. Neural Inf. Process. Syst., 2002.

[20] G. Irie, Z. Li, X.-M. Wu, and S.-F. Chang. Locally linear hashing for extracting non-linear manifolds. In Proc. IEEE Conf. Comp. Vis. Patt. Recogn., 2014.

[21] H. Jégou, M. Douze, C. Schmid, and P. Pérez. Aggregating local descriptors into a compact image representation. In Proc. IEEE Conf. Comp. Vis. Patt. Recogn., pages 3304-3311, 2010.

[22] A. Joly and O. Buisson. Random maximum margin hashing. In Proc. IEEE Conf. Comp. Vis. Patt. Recogn., 2011.

[23] B. Kulis and T. Darrell. Learning to hash with binary reconstructive embeddings. In Proc. Adv. Neural Inf. Process. Syst., 2009.

[24] B. Kulis and K. Grauman. Kernelized locality-sensitive hashing for scalable image search. In Proc. IEEE Int. Conf. Comp. Vis., 2009.

[25] B. Kulis, P. Jain, and K. Grauman. Fast similarity search for learned metrics. IEEE Trans. Pattern Anal. Mach. Intell., pages 2143-2157, 2009.

[26] S. Lafon and A. Lee. Diffusion maps and coarse-graining: A unified framework for dimensionality reduction, graph partitioning, and data set parameterization. IEEE Trans. Pattern Anal. Mach. Intell., 28(9):1393$1403,2006$.

[27] X. Li, G. Lin, C. Shen, A. van den Hengel, and A. Dick. Learning hash functions using column generation. In Proc. Int. Conf. Mach. Learn., 2013.

[28] G. Lin, C. Shen, Q. Shi, A. van den Hengel, and D. Suter. Fast supervised hashing with decision trees for high-dimensional data. In Proc. IEEE Conf. Comp. Vis. Patt. Recogn., Columbus, Ohio, USA, 2014.

[29] J. Liu, Z. Huang, H. Cai, H. T. Shen, C. W. Ngo, and W. Wang. Near-duplicate video retrieval: Current research and future trends. $A C M$ Computing Surveys, 45(4):44, 2013.

[30] W. Liu, J. Wang, R. Ji, Y. Jiang, and S. Chang. Supervised hashing with kernels. In Proc. IEEE Conf. Comp. Vis. Patt. Recogn., 2012.

[31] W. Liu, J. Wang, S. Kumar, and S.-F. Chang. Hashing with graphs. In Proc. Int. Conf. Mach. Learn., 2011.

[32] Y. Liu, F. Wu, Y. Yang, Y. Zhuang, and A. G. Hauptmann. Spline regression hashing for fast image search. IEEE Trans. Image Proc. 21(10):4480-4491, 2012.

[33] C. D. Manning, P. Raghavan, and H. Schtze. Introduction to Information Retrieval. Cambridge University Press, New York, NY, USA, 2008

[34] A. Oliva and A. Torralba. Modeling the shape of the scene: A holistic representation of the spatial envelope. Int. J. Comp. Vis., 2001

[35] M. Raginsky and S. Lazebnik. Locality-sensitive binary codes from shift-invariant kernels. In Proc. Adv. Neural Inf. Process. Syst., 2009.

[36] S. Roweis and L. Saul. Nonlinear dimensionality reduction by locally linear embedding. Science, pages 2323-2326, 2000.

[37] F. Shen, C. Shen, Q. Shi, A. van den Hengel, and Z. Tang. Inductive hashing on manifolds. In Proc. IEEE Conf. Comp. Vis. Patt. Recogn., 2013.

[38] A. Talwalkar, S. Kumar, and H. A. Rowley. Large-scale manifold learning. In Proc. IEEE Conf. Comp. Vis. Patt. Recogn., 2008.
[39] J. Tenenbaum, V. De Silva, and J. Langford. A global geometric framework for nonlinear dimensionality reduction. Science, pages 2319 2323, 2000.

[40] A. Torralba, R. Fergus, and W. Freeman. 80 million tiny images: A large data set for nonparametric object and scene recognition. IEEE Trans. Pattern Anal. Mach. Intell., pages 1958-1970, 2008.

[41] L. Van der Maaten and G. Hinton. Visualizing data using t-SNE. $J$. Mach. Learn. Res., 9:2579-2605, 2008.

[42] J. Venna, J. Peltonen, K. Nybo, H. Aidos, and S. Kaski. Information retrieval perspective to nonlinear dimensionality reduction for data visualization. J. Mach. Learn. Res., 11:451-490, 2010.

[43] J. Wang, S. Kumar, and S.-F. Chang. Semi-supervised hashing for large scale search. IEEE Trans. Pattern Anal. Mach. Intell., 34(12):2393 2406, 2012.

[44] J. Wang, J. Yang, K. Yu, F. Lv, T. Huang, and Y. Gong. Localityconstrained linear coding for image classification. In Proc. IEEE Conf. Comp. Vis. Patt. Recogn., 2010.

[45] Y. Weiss, R. Fergus, and A. Torralba. Multidimensional spectral hashing. In Proc. Eur. Conf. Comp. Vis., pages 340-353, 2012.

[46] Y. Weiss, A. Torralba, and R. Fergus. Spectral hashing. In Proc. Adv. Neural Inf. Process. Syst., 2008.

[47] K. Yu, T. Zhang, and Y. Gong. Nonlinear learning using local coordinate coding. In Proc. Adv. Neural Inf. Process. Syst., 2009.

[48] D. Zhang, J. Wang, D. Cai, and J. Lu. Self-taught hashing for fast similarity search. In Proc. ACM SIGIR Conf., 2010. 